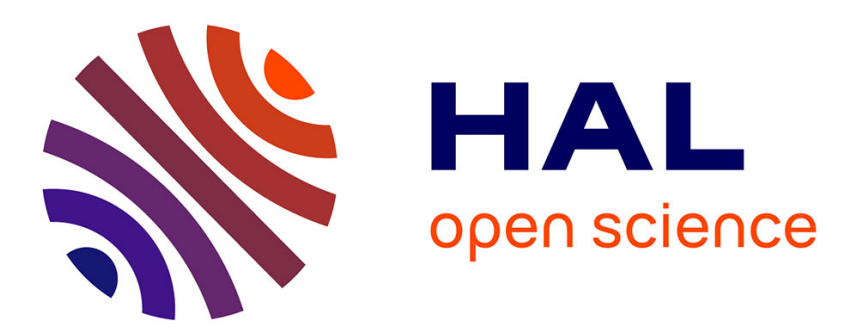

\title{
Utilisation des critères non financiers pour évaluer ou piloter la performance: analyse théorique
}

Grégory Wegmann, Evelyne Poincelot

\section{To cite this version:}

Grégory Wegmann, Evelyne Poincelot. Utilisation des critères non financiers pour évaluer ou piloter la performance : analyse théorique. Comptabilité - Contrôle - Audit, 2005, 11 (2), pp.109-125. halshs00481768

\section{HAL Id: halshs-00481768 \\ https://shs.hal.science/halshs-00481768}

Submitted on 9 May 2010

HAL is a multi-disciplinary open access archive for the deposit and dissemination of scientific research documents, whether they are published or not. The documents may come from teaching and research institutions in France or abroad, or from public or private research centers.
L'archive ouverte pluridisciplinaire HAL, est destinée au dépôt et à la diffusion de documents scientifiques de niveau recherche, publiés ou non, émanant des établissements d'enseignement et de recherche français ou étrangers, des laboratoires publics ou privés. 


\title{
Utilisation des critères non financiers pour évaluer ou piloter la
}

\section{performance : analyse théorique}

\author{
Evelyne Poincelot*, \\ Maître de conférences en Sciences de Gestion, HDR, IAE de Dijon / LEG \\ Grégory Wegmann, \\ Maître de conférences en Sciences de Gestion, IAE de Dijon / LEG
}

Septembre 2004

Cahiers du Fargo : $n^{\circ} 1040902$

\section{Résumé :}

Les déterminants justifiant l'utilisation des critères non financiers pour évaluer ou piloter la performance renvoient à des explications théoriques en liaison avec les approches contractuelles et cognitives. L'objectif de notre article est de proposer pour ces deux approches une analyse théorique. Pour l'approche contractuelle, nous dressons une typologie des explications du choix des critères non financiers avancées dans les travaux existants. Nous montrons également que d'autres théories auraient pu être mobilisées. Pour l'approche cognitive, nous précisons les travaux qui établissent explicitement un lien entre une lecture théorique et les critères non financiers ainsi que d'autres travaux pour lesquels nous établissons ce lien. Nous précisons enfin le référentiel théorique auquel peuvent se rattacher des outils présentant de nombreux critères non financiers comme le Balanced Scorecard ou le Capital Intellectuel.

Mots clés : Critères non financiers - Théories contractuelles - Théories cognitives - Contrôle - Performance.

\begin{abstract}
The Use of Non-financial Measures to Evaluate or Manage the Performance: A Theoretical Analysis
\end{abstract}

The determinants which explain the use of non-financial measures in order to evaluate or drive the performance belong to disciplinary (contractual) and knowledge-based approaches. The aim of this paper is to propose a theoretical analysis of these explanations. For the disciplinary view, we realize a typology of the existing literature. We show that others theories explain the choice of non-financial measures too. For the knowledge-based view, we propose a synthesis of the existing literature in which the link between non-financial and theoretical explanations exists or in which we highlight it. Finally, we explain why the Balanced Scorecard can be associated to the disciplinary view and the Intellectual Capital to the knowledge-based view.

Keywords: Non-financial measures Disciplinary theories - Knowledge-based theories - Control - Performance

\footnotetext{
* Correspondance : Institut d'Administration des Entreprises, Pôle d'Economie et de Gestion - BP 26611 21066 Dijon Cedex evelyne.poincelot@u-bourgogne.fr.
} 


\section{Introduction}

Les déterminants justifiant l'utilisation des critères non financiers ${ }^{1}$ pour évaluer ou piloter la performance sont multiples et renvoient à des explications théoriques diverses. L'intérêt que nous portons à ces critères se justifie dans un contexte actuel (scandales financiers, volatilité accrue des marchés...) montrant les limites de l'information comptable et financière comme vecteur de communication de la performance. En parallèle, des mesures financières ont rencontré un regain d'intérêt à travers par exemple la valeur ajoutée économique (traduction de l'EVA) et des mesures non financières ont été introduites dans des outils comme par exemple, le Balanced Scorecard. Quelles sont en définitive les justifications à mettre en place des indicateurs non financiers ? Ne sontils par exemple qu'un vecteur de communication interne ou externe? Nous tenterons d'apporter des éléments de réponse à ces questions en nous focalisant sur les explications supposant que les critères non financiers sont utilisés dans le but de créer de la valeur².

Pour les théories contractuelles, la clé de la performance est liée à la structuration du système de contrôle et à l'allocation des droits de propriété (Charreaux, 2002 a, p.30). Par exemple, selon la théorie de l'agence (Jensen et Meckling, 1976), les fonctions de propriété comprennent une fonction décisionnelle (du ressort des managers) et une fonction de contrôle qui renferme les systèmes d'incitation et de contrôle mis en place par les actionnaires. Les conflits d'intérêts entre actionnaires et dirigeants proviennent d'une délégation du pouvoir décisionnel des actionnaires aux dirigeants. La recherche de la minimisation des coûts liés à ces conflits d'intérêts résultant du démembrement de ces droits est fondamentale et la source de l'efficience est essentiellement disciplinaire. Dans la théorie de l'architecture organisationnelle (Jensen et Meckling, 1992), la fonction décisionnelle est partagée entre les dirigeants et leurs subordonnés et les conflits d'intérêts entre eux naissent de cette 
délégation. Les dirigeants sont amenés à mettre en place des systèmes d'incitation et de contrôle pour discipliner les salariés.

Dans ce cadre, les critères non financiers s'insèrent dans les mécanismes incitatifs et de contrôle afin d'éviter que le dirigeant gaspille de la valeur potentielle. Ils permettent de réduire les conflits et ceci davantage que les critères financiers, car ils sont aussi des moyens plus adaptés pour évaluer la performance. Ils s'insèrent également dans les mécanismes incitatifs et de contrôle des salariés afin d'éviter un gaspillage de la valeur potentielle.

Pour les théories cognitives (théories fondées sur la connaissance), la performance est liée au fait pour une organisation de savoir s'adapter à son environnement et aussi de savoir développer un stock de connaissances susceptible de créer de la valeur (Charreaux, 2002 a). Les critères non financiers n'ont alors plus comme but ultime de limiter les conflits, mais plutôt d'éclairer le management sur la façon d'atteindre ce double objectif : s'adapter et apprendre. Celui-ci renvoie à des dimensions à la fois sociologiques et psychologiques du pilotage des performances. C'est pourquoi ces critères reflètent une diversité des points de vue et le caractère équivoque de la prise de décision en management. Cette conception a de fortes incidences sur la façon d'appréhender les outils de pilotage des organisations dans lesquels s'insèrent les critères non financiers; non plus des outils formels qui véhiculent des analyses exhaustives et orientent les décisions dans des directions précises, mais des dispositifs flous, non exhaustifs (logique de pilotage par les priorités), mêlant aspects formels et informels, non exhaustifs et qui laissent la place aux ajustements cognitifs.

L'objectif de notre article est de proposer pour chaque approche retenue (contractuelle et cognitive), une analyse théorique. Pour l'approche contractuelle, nous dressons une typologie des explications du choix des critères non financiers avancées dans les travaux existants. Nous montrons également que d'autres théories auraient pu être mobilisées. Pour l'approche cognitive, nous précisons les travaux qui établissent explicitement un lien entre lecture théorique et critères non financiers ainsi que d'autres travaux pour lesquels nous établissons ce lien. Nous précisons également le référentiel théorique auquel peuvent se rattacher des outils présentant de nombreux critères non financiers 
comme le Balanced Scorecard ou le Capital Intellectuel. Nous concluons ensuite en évoquant les divergences constatées entre les approches contractuelle et cognitive et nous présentons des pistes d'approfondissement.

\section{Approches contractuelles : analyse théorique}

\section{Taxonomie des théories contractuelles et explications justifiant l'utilisation des critères non financiers pour apprécier la performance d'une organisation}

En réalisant une synthèse des approches contractuelles, nous constatons que les critères non financiers atténuent les conflits d'intérêts entre dirigeants et salariés ou entre actionnaires et dirigeants en améliorant la connaissance des efforts accomplis ou des résultats obtenus par les agents dans la réalisation de leurs contrats.

Par théories contractuelles, nous entendons un ensemble de grilles de lectures dérivées de la théorie économique néoclassique. Ces théories ont pour caractéristique majeure de considérer des variables disciplinaires comme les déterminants fondamentaux de la création de valeur dans une organisation. Parmi ces théories contractuelles nous pouvons citer, entre autres, la théorie de l'agence et de l'architecture organisationnelle.

Dans une approche contractuelle, les différents systèmes de mesure des performances, en particulier en introduisant des critères non financiers, ont pour fonctions de faire correspondre la stratégie à l'allocation des droits décisionnels d'une organisation, d'assurer la cohérence interne de cette organisation, de jouer un rôle informationnel et ainsi, en évitant de détruire de la valeur, de réduire les conflits d'intérêts.

\subsection{Les critères non financiers d'évaluation des performances facilitent la cohérence entre la stratégie et l'allocation des droits décisionnels}


Les différents systèmes de mesure des performances, en particulier les critères non financiers, aident en premier lieu les managers à assurer la cohérence entre la stratégie et l'allocation des droits décisionnels.

De nombreuses recherches s'inscrivant dans le courant conventionnel de la stratégie ${ }^{3}$ expliquent le recours à des critères non financiers sur cette base. La figure 1 (Brikley et al., 1997), qui prend appui sur une lecture stratégique, montre l'articulation qui existe entre des variables stratégiques et la structure organisationnelle (définie par l'allocation des droits décisionnels, les systèmes d'incitation, de contrôle et d'évaluation de la performance). 


\section{Schéma 1.}

Stratégie, allocation des droits décisionnels et critères non financiers

(Brikley et al. (1997 p.179))

\begin{tabular}{|c|c|c|}
\hline \multicolumn{2}{|c|}{ Critères définissant le caractère fluctuant de l'environnement de l'entreprise } \\
\hline «technologique » (affectant les \\
Eroduits, les méthodes de \\
production et les systèmes \\
d'information)
\end{tabular}

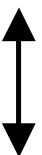

Architecture organisationnelle

Cohérence entre l'allocation des droits décisionnels et les systèmes de contrôle (dont le système d'évaluation de la performance) 
Cette recherche de cohérence est un rôle qui a traditionnellement été dévolu aux dispositifs de contrôle de gestion (Anthony, 1965). Mais ces dispositifs reposaient pour l'essentiel sur des critères de mesures financières, qu'il s'agisse de dispositifs de calcul de coûts ou de gestion budgétaire. L'idée que des critères non financiers puissent jouer un rôle non négligeable dans cette recherche de cohérence est assez récente. Johnson et Kaplan (Chapitre 11: «Des systèmes de mesure des performances pour le futur, 1987) avancent l'idée, à l'issue d'un constat très sévère sur l'état du contrôle de gestion, que les critères non financiers peuvent faciliter la déclinaison de la stratégie d'une organisation et la validation de ses choix stratégiques, c'est-à-dire garantir la cohérence entre la stratégie et l'allocation des droits décisionnels.

De nombreux travaux sont venus par la suite confirmer cette intuition. La présence de critères non financiers facilite cette cohérence, soit en jouant le rôle de mécanisme incitatif (Ittner et Larcker (2002) et Chenhall (1997)), soit comme composant essentiel du système de contrôle (Ittner et Larcker (1997)), soit les deux à la fois (Fullerton et McWatters (2002)), soit enfin comme moyen de mieux évaluer la performance réalisée (Perera, Harrison et Poole (1997)). Ces derniers notent (pp. 561 et $569,1997)$ la nécessité d'inciter les subordonnés à concentrer leurs efforts vers les priorités stratégiques de l'organisation. Lorsque ces priorités sont orientées vers la satisfaction des clients, les critères non financiers permettent de mieux évaluer la performance des subordonnés. Ittner et Larcker (1997), Govindarajan et Gupta (1985), Fullerton et McWatters (2002) appuient leurs démonstrations relatives à la cohérence entre la stratégie et l'architecture organisationnelle en étudiant des entreprises qui développent respectivement des stratégies orientées vers la qualité, la différenciation ou le déploiement en juste à temps. Ces critères non financiers seraient utilisés à différents niveaux hiérarchiques (personnels d'encadrement (Govindarajan et Gupta (1985) ou exécutifs (Ittner et Larcker (2002)).

Les différents travaux que nous avons étudiés montrent que les critères non financiers sont en définitive prépondérants lorsque la fonction décisionnelle est décentralisée. Ils constituent alors un mécanisme d'incitation, de contrôle et d'évaluation des performances, plus adapté que les critères 
financiers. En effet, selon Brikley et al (1997, p.178), un mouvement de décentralisation dans une organisation peut s'expliquer par une augmentation de l'incertitude de l'environnement, une stratégie prix, ou bien orientée vers l'amélioration de la qualité ou de la satisfaction des clients. L'utilisation de critères non financiers accompagne ce mouvement.

\subsection{L'utilisation de critères non financiers peut favoriser la cohérence interne de l'architecture organisationnelle}

Dans une logique contractuelle, une cohérence accrue entre l'allocation des droits décisionnels et les systèmes de contrôle (systèmes d'évaluation de la performance, d'incitation et de sanction) est source de création de valeur pour l'organisation. Assurer un niveau optimal de délégation de la prise de décision afin de minimiser les coûts liés à une insuffisance d'informations pertinentes, à des comportements déviants de la part des agents et à un manque de coordination constitue la clé de la performance (Jensen et Meckling, 1992, p.115).

Selon la Théorie de l'Architecture Organisationnelle, le choix de la structure et des critères de performance associés est fonction du risque de comportements déviants des salariés s'ils détiennent des droits décisionnels. C'est pourquoi la structure d'une organisation en centres de responsabilités conditionne les règles du choix des critères de performance et du système incitatif et de contrôle (Jensen et Meckling, 1992, p.121). Ces critères sont différents selon qu'il s'agisse d'une structure en centres de recettes, de coût ou encore de profit. Cette structuration dépend bien évidemment de la nature de la connaissance nécessaire aux prises de décision dans les différents centres ainsi qu'au siège. Prenons un exemple. Selon Jensen et Meckling (1998), si la connaissance concernant la qualité ou la mesure de la quantité optimale est difficile à appréhender par les dirigeants, ceux-ci éviteront de transformer l'unité de production en un centre de coût. En effet, l'objectif assigné au responsable d'un centre de coût, à savoir minimiser le coût total d'une quantité de produit, sera en fait réduit en un objectif de minimisation de coût moyen unitaire, si le système incitatif est fondé sur le respect du 
second objectif, c'est-à-dire la quantité optimale. Ces deux objectifs seraient équivalents si le responsable d'unité détermine une quantité à produire maximisant la valeur de la firme (quantité optimale). Si la quantité est différente et s'il détient une latitude décisionnelle dans sa détermination, la performance de la firme en sera affectée. Autrement dit, l'objectif consistant à réduire le coût unitaire des produits peut se faire au détriment de la quantité optimale ou de la qualité. Si la qualité est facilement observable, on conserve un centre de coût. Dans le cas contraire, on choisit un centre de profit. Ainsi, nous en déduisons que des indicateurs non financiers concernant la qualité des produits et le degré de satisfaction du client se retrouveront plus facilement dans une organisation présentant des centres de coût, c'est-à-dire a priori plus centralisée qu'une structure en centre de profit.

Mais nous pouvons estimer à l'inverse qu'un fort niveau de décentralisation nécessite de multiplier les variables de contrôle et de pilotage, ce qui inciteraient aussi à l'utilisation d'indicateurs diversifiés et notamment non financiers. Cette dernière réflexion est-elle contradictoire avec l'analyse faite par Jensen et Meckling ? Oui, si nous supposons que le degré de décentralisation de la prise de décision est systématiquement moins important pour un centre de coût que pour un centre de profit et, par extension, que le degré de décentralisation caractérise le découpage en centres de responsabilité. Mais cela n'est pas toujours le cas. Selon Horngern et al. (2003 p.199), «les responsables d'une division organisée en centre de profit peuvent n'avoir qu'une faible marge d'initiative. D'autres divisions peuvent être organisées en centre de coûts alors que leurs responsables disposent d'une grande latitude pour choisir leur fournisseur ».

Nos derniers développements montrent que dans la littérature contractuelle, les relations entre l'architecture organisationnelle et les critères non financiers ne sont pas clairement établies. 


\subsection{La présence d'indicateurs non financiers dans les contrats incitatifs du dirigeant comme moyen de réduction des conflits}

Les critères non financiers peuvent dans certains cas se révéler plus adaptés aux mécanismes d'incitation et de contrôle. Ittner, Larcker et Rajan (1997), s'inspirant notamment des travaux de Feltham et Xie (1994), considèrent que les contrats incitatifs managériaux fondés uniquement sur des mesures financières de la performance ne sont pas le moyen le plus efficient de motiver les dirigeants à agir conformément aux intérêts des actionnaires. Ils doivent être complétés par des critères non financiers qui informent davantage et plus rapidement les actionnaires des efforts accomplis par les dirigeants pour créer de la valeur. Ces critères s'avèrent tout particulièrement pertinents lorsque les dirigeants choisissent par exemple une stratégie de développement de nouveaux produits, d'augmentation des parts de marché ou bien encore une stratégie orientée qualité ou si la firme est dans une situation de détresse financière. La présence de ces indicateurs est alors un moyen de réduire les conflits opposant dirigeants et actionnaires. Ittner, Larcker et Rajan (1997) considèrent également que des indicateurs non financiers peuvent être davantage sujets à des manipulations managériales, difficilement décelables, ce qui conduirait à un effet contre-productif. Ils testent donc la pertinence d'introduire des indicateurs non financiers dans les contrats de bonus proposés aux dirigeants et concluent qu'ils joueraient plutôt un rôle informationnel. Ce rôle est également retenu lorsque le système incitatif concerne les salariés exécutants (Ittner et Larcker (2002)).

D'autres théories peuvent être mobilisées afin d'expliquer la présence d'indicateurs non financiers. Pour la théorie fondée sur les contrats implicites (Cornell et Shapiro, 1987), la création de la valeur est liée à la création du capital organisationnel (surplus spécifique associé à la mise en œuvre de contrats implicites noués avec des stakeholders). Un contrat implicite serait par exemple pour une entreprise de faire des efforts pour accroître la durée d'utilisation de ses produits via une plus grande qualité et la créance implicite serait le supplément de prix que la clientèle accepterait de payer. Des indicateurs non financiers axés dans ce cas sur la satisfaction de la clientèle seraient un moyen de témoigner de ces efforts. Dans une perspective de répartition de la valeur créée par une organisation 
entre, non pas seulement les actionnaires, mais entre les stakeholders (les parties prenantes aux activités de cette organisation), les dirigeants ont intérêt à favoriser (ou à défavoriser) les stakeholders qui contribuent le plus (ou le moins) à la création de valeur. La répartition de la valeur conditionne la valeur créée. Ce raisonnement peut conduire ces dirigeants à rémunérer certains stakeholders au-delà (ou en deçà) de leurs coûts d'opportunité (Charreaux et Desbrières (1998)). D'Souza et Williams (2000, p.234) suggèrent une corrélation entre la nature des indicateurs de performance, leur évolution et l'importance des stakeholdersclés dans l'activité de l'entreprise, sans toutefois détailler la nature de ces indicateurs. Dans une approche stakeholders, une réflexion préalable s'impose afin d'identifier, selon les stakeholders, les facteurs justifiant qu'ils sont plus ou moins importants dans le processus de création de valeur. Pour les salariés, quels sont les facteurs qui justifieraient une rémunération différente du coût d'opportunité ? Prenons l'exemple d'une entreprise qui déploie une stratégie qui conduit à ce que des compétences clés jouent un rôle déterminant dans la création de valeur. Dans une telle situation, nous pouvons penser que des critères non financiers relatifs par exemple à la fidélisation des salariés ou encore à leur degré de motivation joueront un rôle déterminant. De la même façon, des indicateurs de performance concernant les clients (un indicateur de satisfaction par exemple) seront davantage présents si l'entreprise a mené une stratégie de différenciation fondée sur l'innovation.

\subsection{Un dispositif fondé sur des indicateurs non financiers élaboré dans une perspective contractuelle : le Balanced Scorecard}

Kaplan et Norton $(1998,2004)$, à l'origine du Balanced Scorecard ${ }^{4}$ ne proposent pas de lecture théorique de leur dispositif de pilotage. Rappelons qu'un Balanced Scorecard est un tableau de bord où des indicateurs sont articulés à un certain nombre d'objectifs stratégiques définis au préalable. Ces indicateurs sont pour bon nombre d'entre eux de nature non financière. Plusieurs éléments confirment que le Balanced Scorecard s'inscrit dans une perspective contractuelle. 
Les perspectives et les objectifs stratégiques sont prédéterminés. C'est pourquoi les indicateurs ont pour objectifs de décliner la stratégie et de valider les hypothèses stratégiques formulées $(2004$, p. 32). Cette démarche d'alignement stratégique s'établit au travers de ce que Kaplan et Norton intitulent des Cartes Stratégiques.

Cette méthode s'appuie sur un modèle stratégique de type concurrentiel (Porter, 1985) : les opportunités et les menaces de l'environnement confrontées aux forces et aux faiblesses de l'entreprise déterminent les stratégies fonctionnelles à déployer (différenciation, domination par les coûts ou niche). Cet outil semble s'inscrire dans une logique de coûts de dédouanement du dirigeant dans la relation d'agence le liant aux actionnaires.

Pour Kaplan et Norton (1996), les acteurs principaux de la mise en œuvre d'un Balanced Scorecard sont des consultants et les dirigeants de l'entreprise. La participation des opérationnels n'est pas requise. Il s'agit d'un dispositif mis à la disposition de la direction générale. Il est bien souvent adossé à des systèmes de rémunération individuelle au mérite (Bourguignon et al. (2002)). Le caractère «prédéterminé » et l'objectif d'évaluer (plus que de piloter) la performance limitent le processus d'apprentissage et de création de connaissance.

Nous conclurons cette première partie en faisant ressortir les points communs des approches contractuelles. Premièrement, les conséquences liées à l'introduction des indicateurs non financiers sont prédéterminées et la valeur résultante de leur application est créée rapidement. Deuxièmement, une forte décentralisation de la fonction décisionnelle est un déterminant important justifiant leur introduction. Il en résulte qu'ils sont destinés à ceux qui souhaitent être rassurés quant à l'exécution optimale des contrats. Enfin, la dimension concernant l'évaluation de la performance est prépondérante : les indicateurs non financiers seraient principalement un instrument de mesure de la valeur créée. 


\section{Approches cognitives : analyse théorique}

Comme le montre Charreaux (p. 25, 2002 b), des approches en rupture avec le paradigme contractuel peuvent être regroupées sous l'appellation «théories cognitives de la firme ». D'après Charreaux, ces théories regroupent notamment le courant comportemental (Simon, 1947 ; Cyert et March, 1963), la théorie évolutionniste (Nelson et Winter, 1982), et la théorie fondée sur les ressources et les compétences (Penrose, 1959).

Après avoir caractérisé brièvement l'approche cognitive, notre objectif est d'évoquer les travaux faisant explicitement un lien entre l'une de ces théories et la présence des indicateurs non financiers. Ces travaux sont focalisés généralement sur le concept de Capital Intellectuel (Intellectual Capital ${ }^{5}$ ), expression détaillée du goodwill. Notre objectif est également de justifier la présence de ces indicateurs à partir de cette approche.

\subsection{Présentation des théories cognitives et liens avec les indicateurs non financiers}

Le point commun aux théories cognitives est que la création de valeur provient de la connaissance. La source de la création de valeur est liée à des éléments difficilement imitables qui procurent ainsi un avantage concurrentiel significatif et durable. Les déterminants de la création de valeur que l'on recense dans les théories cognitives sont pour certains de nature sociologique et pour d'autres de nature psychologique. Granovetter (1994), en expliquant que toute organisation est encastrée dans un cadre social (notion d'embeddedness), met en lumière la dimension sociologique de ces théories.

La théorie comportementale (théorie behavioriste) met davantage en évidence les dimensions psychologiques de la création de valeur. L'atteinte de la performance souhaitée nécessite de connaître les comportements des acteurs ou des groupes d'acteurs d'une organisation. Deux hypothèses sont à la base de cette théorie : la rationalité des individus est limitée (Simon, 1947) et l'organisation est 
constituée d'une coalition d'acteurs aux objectifs propres (March et Simon, 1958), ce qui est source de divergences et de conflits potentiels. En conséquence, un principe de maximisation de la satisfaction se substitue au principe traditionnel d'optimisation de la valeur pour les actionnaires. Le processus de décision est interactif et émergent et donne la possibilité d'apprentissages organisationnels.

Les dispositifs de contrôle ont dans ce contexte pour objectif de garantir la pérennisation de l'organisation. Pour cela, le management doit remplir une mission d'animation et de médiation afin d'aider les acteurs à se coordonner. Ces dispositifs doivent également informer sur les niveaux de contributions des différents acteurs et de la sorte sur les rétributions envisageables, et permettre ainsi une réduction du «slack organisationnel ». Pour parvenir à ces fins, le contrôleur doit mesurer et transmettre des signaux internes et externes, stabiliser les comportements et favoriser l'autocontrôle (Langevin, 1999). Des indicateurs non financiers (taux d'absentéisme, indicateur de productivité...) renseignant sur le comportement de chacun peuvent augmenter le ciment de cette coalition.

Dans les théories de l'apprentissage organisationnel, la compétence créant de la valeur provient de la connaissance des routines organisationnelles et plus particulièrement pour la théorie évolutionniste (Nelson et Winter, 1982), de la connaissance de leurs évolutions. Les routines sont des modèles de comportements et d'interactions que les individus sont capables d'utiliser pour faire face aux différentes situations qui se présentent. La construction de ces routines correspond à l'apprentissage organisationnel (collectif). Elles sont généralement tacites (donc ni codifiables, ni transférables). Nonaka et Takeuchi (1997) expliquent la création de connaissances organisationnelles ${ }^{6}$ et distinguent les apprentissages organisationnels à dominante comportementale (apprendre «comment faire » par observation, imitation, expérience) et à dominante cognitive (« les résultats de l'apprentissage sont un changement cognitif qui se traduit par un traitement d'informations et entraîne un enrichissement des connaissances ou une modification des schémas d'interprétation» (p.7). Les processus d'apprentissage concerneront alors les «savoir quoi» et les «savoir pourquoi»). Un processus d'apprentissage organisationnel complet entraîne un changement comportemental et cognitif. Nonaka et Takeuchi (1997) considèrent les conditions organisationnelles favorables à cet apprentissage et 
notamment précisent «qu'un accès rapide à une information variée et large, des structures d'organisation flexibles et dont les unités sont reliées, des changements fréquents dans ces structures et des rotations de postes (p.13)» sont autant de facteurs qui contribuent à faire partager et émerger la connaissance, y compris tacite entre les individus et en définitive à créer de la valeur. La pluralité des indicateurs non financiers et leur fréquence élevée incluant ceux axés sur la formation, le taux de rotation du personnel peuvent se justifier dans cette théorie afin de susciter l'apprentissage organisationnel. La participation des autres stakeholders à la connaissance des salariés est importante également (opinions des clients sur les produits, les services...). Notons également que la création de la connaissance organisationnelle se fait initialement en transférant des connaissances tacites au sein d'un groupe, en partageant de l'information. La nature des informations à communiquer n'est pas dictée par la hiérarchie mais émerge de la communication entre les groupes (les connaissances tacites étant par essence difficilement transférables, leur transfert se fera surtout au travers d'un dialogue et moins de façon formalisée au travers d'indicateurs).

Dans la théorie évolutionniste, l'évolution d'une organisation s'explique par une exploitation pertinente de compétences dites secondaires. Langevin (1999, p. 211), donne l'exemple d'une entreprise au départ industrielle qui a pu se constituer un réseau de distribution qui représente ultérieurement un «actif complémentaire » susceptible de lui donner un avantage compétitif pour d'autres activités. La théorie évolutionniste peut être utilisée aussi pour comprendre que des indicateurs non financiers (comme d'autres pratiques managériales, par exemple la méthode $\mathrm{ABC}$ ) puissent être introduits et conservés ou au contraire rapidement rejetés en étudiant les routines organisationnelles existantes (Burns et Scapens, 2000).

Le Mouvement des Ressources et Compétences (désormais MRC; Tywoniak, in Laroche et Nioche, 1998, p. 166) trouve son origine dans les travaux de Ricardo (Arrègle, 1996, p. 25) à l'occasion desquels ont été développées les notions de Rente et de Quasi-rente. La Rente ricardienne consiste en la possession et l'utilisation d'un actif stratégique rare dont l'offre est limitée et qui ne peut pas être facilement imité ou créé. La quasi-rente est la spécificité d'un actif qui pourra être d'une 
valeur supérieure pour une entreprise que pour un concurrent. Ces actifs sont difficilement imitables, difficilement substituables et échangeables sur un marché. Le MRC, s'inscrit dans le cadre plus large des théories évolutionnistes qui postulent que la dimension structurante de la performance des entreprises n'est pas leur position concurrentielle, mais la gestion de l'évolution de leurs procédés techniques et de leurs processus organisationnels. Le MRC aboutit donc à recentrer la réflexion stratégique au cœur de l'entreprise en tentant d'identifier ses ressources rares et plus spécifiquement ses compétences humaines et organisationnelles, c'est-à-dire son Capital Intellectuel. Les éléments du Capital Intellectuel sont alors perçus comme des ressources stratégiques de premier ordre, susceptibles de procurer aux entreprises un avantage concurrentiel déterminant. Le MRC renvoie à un mode stratégique interactionniste qui consiste à identifier les ressources et les compétences, puis à analyser l'interaction entre ces ressources et compétences et les conditions de l'environnement.

\subsection{Perspectives cognitives et théories en contrôle organisationnel}

D’une façon générale, les théories cognitives s'appuient sur des modes de contrôle émergents. Ces modes de contrôle ont pour ambition de coordonner les routines. Il s'agit aussi de favoriser l'émergence de ces compétences secondaires en proposant des dispositifs de détection et d'analyse adéquats (notion d'indicateurs avancés) et en facilitant l'apprentissage organisationnel : favoriser les échanges, la communication et la formation. Au-delà de cette présentation générale, il est intéressant de montrer que certaines théories en contrôle organisationnel, c'est-à-dire des théories relatives à l'appréciation des performances et au pilotage des organisations, s'inscrivent dans la perspective cognitive. Nous présentons deux approches particulièrement significatives.

Ouchi (1979) montre qu'en situation d'incertitude, l'entreprise doit faire appel à d'autres modalités de contrôle que le contrôle de nature disciplinaire (vérification que les résultats sont à la hauteur des objectifs fixés et que les comportements sont en accord avec les prescriptions des managers). Pour compléter des pratiques disciplinaires de contrôle, Ouchi préconise en dernier ressort 
des mécanismes informels de contrôle. Il peut s'agir de contrôles claniques ou par les rituels, c'est-àdire de modalités de contrôle fondées par exemple sur la culture d'entreprise ou encore le degré d'autonomie d'un groupe de travail et son niveau de responsabilisation et d'implication (notion d'autocontrôle). Ces modalités impliquent une imbrication inextricable entre des aspects économicostratégiques et des aspects humains.

Dans la figure 2, les modes de contrôles par les résultats et les comportements renvoient au paradigme contractuel, tandis que les modes de contrôles informels, par la culture et l'autocontrôle renvoient au paradigme cognitif.

Schéma 2.

Synthèse sur les modalités de contrôle en situation d'incertitude

Problèmes analysables/ Connaissance complète des relations de cause à effet
Problèmes non analysables/

Connaissance incomplète

Peu d'exceptions/
Standards de
performance
cristallisés
Nombreuses
exceptions/
standards
ambigus

\begin{tabular}{|c|c|}
\hline $\begin{array}{c}\text { Contrôle d'exécution } \\
\text { rapproché/Contrôle } \\
\text { administratif } \\
\text { des comportements }\end{array}$ & $\begin{array}{c}\text { Contrôle des } \\
\text { résultats comptables }\end{array}$ \\
\hline $\begin{array}{c}\text { Contrôle étendu } \\
\text { des comportements et } \\
\text { du personnel }\end{array}$ & $\begin{array}{c}\text { Contrôle "ad-hoc"/ } \\
\text { informel/des inputs }\end{array}$ \\
\hline
\end{tabular}

Simons (1995, tableau 1), également en rupture avec les théories orthodoxes du contrôle, développe le concept de contrôle interactif. Il précise que ce mode de contrôle est pertinent dans des domaines d'activités sujets à des changements rapides où les managers sont confrontés à beaucoup d'incertitudes et à des prises de risques importantes. Pour attirer l'attention de l'organisation sur les incertitudes stratégiques, les dirigeants vont sélectionner certains dispositifs de contrôle qu'ils auront à utiliser de façon interactive. En nous inspirant de Langevin (1999) nous proposons une synthèse des modes de contrôle selon les différentes approches théoriques (tableau 2).

Tableau 1.

Contrôle diagnostic / Contrôle interactif (Simons, p. 124, 1995) 


\begin{tabular}{|c|c|c|}
\hline & Contrôle diagnostic & Contrôle interactif \\
\hline Raison d'être & $\begin{array}{c}\text { Donner une motivation et une direction } \\
\text { pour atteindre les objectifs }\end{array}$ & $\begin{array}{c}\text { Stimuler le dialogue et l'apprentissage } \\
\text { organisationnel }\end{array}$ \\
\hline Objectif & Maîtriser les incertitudes & Rechercher la créativité \\
\hline Raisonnement & Déductif & Inductif et intuitif \\
\hline Système & Complexe & Simple \\
\hline Horizon & Passé et présent & Présent et future \\
\hline Cibles & Fixes & Continuellement révisées \\
\hline Feedback & Négatif & Positif \\
\hline Ajustement & Simple boucle & Double boucle d'apprentissage \\
\hline Communication & Dialogue non indispensable & Langage commun requis \\
\hline Rôle du staff & Gardiens des clés & «Facilitateurs » \\
\hline
\end{tabular}

Tableau 2.

Synthèse : Courants théoriques et modes de contrôle (adapté de Langevin, 1999)

\begin{tabular}{|c|c|c|}
\hline & Approches contractuelles & Approches cognitives \\
\hline $\begin{array}{l}\text { Problème nécessitant } \\
\text { l'existence d'un contrôle }\end{array}$ & Opportunisme des acteurs & $\begin{array}{c}\text { Incertitude sur les } \\
\text { comportements des acteurs }\end{array}$ \\
\hline Finalités du contrôle & Incitations au respect des contrats & $\begin{array}{c}\text { Coordination et aide à la } \\
\text { décision }\end{array}$ \\
\hline Apparition du contrôle & Imposé & Émergent \\
\hline Exercice du contrôle & Hiérarchique & Autocontrôle \\
\hline Rôle du contrôleur & $\begin{array}{l}\text { Décliner la stratégie, assurer la } \\
\text { cohérence interne, mesurer les } \\
\text { performances et attribuer les } \\
\text { rétributions }\end{array}$ & $\begin{array}{l}\text { Formaliser et influencer les } \\
\text { comportements, favoriser la } \\
\text { communication, et } \\
\text { l'apprentissage }\end{array}$ \\
\hline
\end{tabular}




\begin{tabular}{|l|c|c|}
\hline Intervention du contrôle par & A posteriori & \\
rapport aux décisions des & & \\
acteurs & & \\
\hline Circulation de l'information & Verticale & Transversale \\
\hline
\end{tabular}

\subsection{Un dispositif à base d'indicateurs non financiers élaboré dans une perspective cognitive : le Capital Intellectuel}

Pour Mourritsen (1998), les indicateurs non financiers, à travers l'utilisation d'outil comme le «Capital Intellectuel »(désormais CI) sont un moyen de mieux appréhender le potentiel de création de valeur. Pour Roos (1998), le CI permet d'identifier et de visualiser la création de valeur « cachée ». La notion de $\mathrm{CI}$ est «polysémique ». Mais pour tous les spécialistes de cette question, le CI renvoie à la capacité d'une organisation à créer de la valeur à partir de son stock de connaissances. Edvinsson et Sullivan (1996) expliquent que le Capital Intellectuel se compose du Capital Humain (connaissances, savoir-faire, expériences des salariés, attitudes et capacités d'innovation et d'apprentissage) et du Capital Structurel $^{7}$ (compétences qui peuvent être valorisées sur le marché). Le Capital Structurel comprend des actifs tangibles, mais surtout des actifs intangibles en relation avec les partenaires commerciaux (c'est le capital relationnel : liste de prospects, réseau de distribution, alliance avec les fournisseurs...), ou en relation avec la structure (systèmes de calcul de coût, processus de fabrication et d'innovation, processus de décision, ...).

Cette définition du CI met l'accent sur des leviers de création de valeur fondés sur des aspects cognitifs. Il s'agit en effet d'encourager la création de capital humain par de nouvelles formes organisationnelles, de nouveaux styles de management ou encore des systèmes incitatifs appropriés. Il s'agit également de favoriser la création d'actifs commercialisables issus du capital humain. Il s'agit enfin d'améliorer la protection des actifs intellectuels. Pour Roos (1998, p. 151) alors que les théories cognitives sont des théories en quête d'outils opérationnels, le CI est une pratique en quête de 
fondements théoriques. La relation entre l'outil et les approches théoriques reste générale et souvent suggérée. Le CI met en avant le concept de connaissances et de compétences, révèle la capacité d'une organisation à s'adapter à un environnement fluctuant parce que sa stratégie est réfléchie en fonction de son socle interne de compétences, et établit une reconnaissance de l'importance du rôle des salariés dans le processus de création de valeur (en mettant en avant leur vision cognitive) (Mouritsen, 1998). Edvinsson et Sullivan (1996) insistent aussi sur la dimension de la protection de la connaissance $^{8}$. Cette approche est cohérente avec les théories cognitives pour lesquelles la création de valeur est essentiellement liée aux éléments difficilement imitables.

Nous constatons parfois un hiatus entre l'ambition affichée et les indicateurs non financiers déployés dans les entreprises qui utilisent un tel dispositif. Il apparaît bien souvent que les principaux indicateurs développés, centrés notamment sur la mesure de la satisfaction des clients ou encore des salariés, pourraient être justifiés par une grille de lecture contractuelle. En outre, peu d'indications relatives au mode de conception des indicateurs non financiers et aux modalités d'apprentissage et de création de connaissances ne sont données.

Pourtant, quelques expériences semblent prometteuses. C'est le cas notamment de l'entreprise d'assurances suédoises Skandia au sein de laquelle a été développé un tableau de bord à base d'indicateurs du CI (Edvinsson et Malone, 1999). L'annexe 2 présente des éléments de ce dispositif de valorisation du CI. Des indicateurs relatifs aux ressources humaines, technologiques et organisationnelles sont considérés comme les déterminants fondamentaux de la création de valeur, comme le suggère le schéma ci-dessous qui répertorie les perspectives principales de ce tableau de bord. Cette méthode se fonde de manière implicite sur la théorie fondée sur les ressources et les compétences. Elle prend notamment appui sur des stratégies basées sur les ressources et les compétences clés (Hamel et Prahalad, 1990) et s'inscrit dans une logique d'émergence. C'est pourquoi, le processus de mise en place est participatif (Roy, 1999); il est incrémental et peu formalisé. Les opérationnels prennent part au processus d'élaboration et les retours d'expériences sont des étapes importantes de ce processus. Des réflexions menées à un niveau opérationnel (sur des indicateurs) ont eu une incidence sur le profil du tableau de bord. De plus, les indicateurs non 
financiers déployés sont susceptibles de faire émerger de nouvelles orientations stratégiques. Le développement de progiciels de gestion interactifs, d'un intranet, ainsi que d'outils de simulation et de partage d'informations (le Dolphin System) ont accompagné le développement des indicateurs non financiers ${ }^{9}$. Ce sont les employés qui, en fonction de leurs compétences, ont la responsabilité d'un certain nombre d'indicateurs. C'est pourquoi cette expérience illustre également la théorie de l'apprentissage organisationnel.

Vaivio (1999) analyse l'émergence d'indicateurs non financiers dans une entreprise ${ }^{10}$ en s'appuyant notamment sur la théorie de l'apprentissage organisationnel (Nonaka et Takeuchi, 1997). Il explique que les mesures non financières n'ont pas comme seul rôle de décliner la stratégie. Elles permettent également de faire émerger de nouvelles stratégies. Il montre que les mesures non financières s'inscrivent progressivement dans la vie de l'organisation. Celles-ci émergent grâce à un enchaînement de processus structurés et formels ${ }^{11}$ (ce qu'il nomme «revolutionary change ») et de processus informels et incrémentaux (« evolutionary change »).

Dans une étude récente (2004) menée dans la même entreprise, Vaivio montre que les indicateurs non financiers rapprochent les managers du terrain, stimulent les échanges et les débats entre opérationnels, provoquent l'émergence d'apprentissages et peuvent être sujets à controverse. En insistant davantage sur les jeux d'acteurs ${ }^{12}$ et sur le rôle primordial des phénomènes d'apprentissage à un niveau très local, il met en avant le caractère «provocateur » que peuvent revêtir les indicateurs non financiers. A la différence des indicateurs financiers, les indicateurs non financiers semblent avoir la capacité de s'inscrire dans la texture la plus intime d'une organisation. C'est pourquoi ces indicateurs combinent de façon inextricable une dimension rationnelle et une dimension émotionnelle. 


\section{Figure 3.}

Les perspectives stratégiques du tableau de bord fondé sur le Capital Intellectuel de Skandia (adapté d'Edvinsson et Malone, 1999)

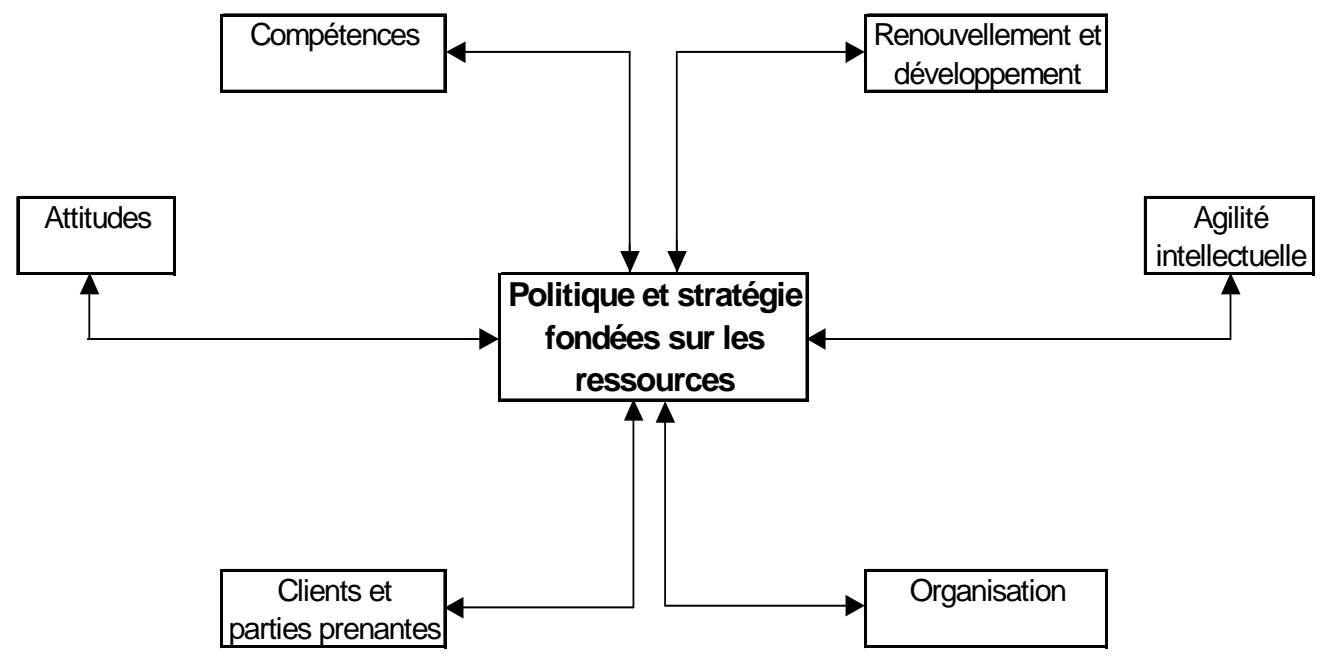

Pour conclure cette seconde partie, nous rappellerons que les approches cognitives sont focalisées sur la justification des indicateurs non financiers pendant leur durée d'utilisation (les conséquences liées à leur introduction émergent pendant leur phase d'utilisation). La création de valeur se « construit». Le point commun à l'ensemble de ces explications serait un partage plus important de la connaissance entre les acteurs (pour une meilleure coordination, un meilleur ajustement mutuel, une plus grande capacité à apprendre...). La connaissance induite est destinée en priorité à ceux qui la font émerger. Enfin, elles privilégient la dimension pilotage.

\section{Conclusion}

Nous constatons de profondes divergences entre les approches contractuelle et cognitive que nous présentons dans le tableau 3. Cinq points de divergences sont retenus, à savoir les destinataires, l'objectif, l'horizon temporel (autrement dit, le délai à l'issue duquel l'introduction de ces indicateurs 
est censée accroître la valeur), la principale condition d'introduction (systématiquement associée à d'autres variables explicatives) et enfin, la nature des conséquences liées à leur utilisation.

Tableau 3.

Principaux points de divergence entre les approches contractuelles et cognitives

\begin{tabular}{|l|c|c|}
\hline & Approches contractuelles & Approches cognitives \\
\hline Destinataire & Les mandants & Les « mandataires » en priorité \\
\hline Objectif principal & Évaluer & Piloter, Coordonner \\
\hline Horizon temporel & Création de valeur immédiate & Création de valeur non \\
& immédiate \\
\hline Principal déterminant & Décentralisation de la fonction & Décentralisation de la \\
& décisionnelle & connaissance \\
\hline Conséquences & Prédéterminées & Non prédéterminées \\
\hline
\end{tabular}

À notre connaissance, il n'existe pas d'études centrées sur des variables qui justifieraient qu'une entreprise choisisse les indicateurs non financiers dans une logique contractuelle plutôt que cognitive (et inversement). Les lectures contractuelles ou cognitives ont en commun de montrer que ces indicateurs sont utilisés comme des instruments au service d'un même objectif : la création de la valeur. Si ces théories sont valides, les entreprises, en cas de cohérence entre la présence des indicateurs non financiers et des variables explicatives justifiant ce choix, devraient être en mesure de dégager une performance supérieure. Ces réflexions seront prolongées par l'étude d'un questionnaire qui nous permettrait 1) de constater les grilles théoriques expliquant le recours aux indicateurs non financiers et 2) de voir pour chacune d'entre elles si une performance supérieure est observée en cas de cohérence entre les variables explicatives supposées et les indicateurs non financiers. Si cette dernière hypothèse n'est pas toujours corroborée, cela montrerait les limites de cette explication théorique. 
Quelques études ont été réalisées afin de tester si la présence des indicateurs non financiers améliore la performance. Mais, chaque test présenté a pour vocation d'infirmer ou non une ou deux explications possibles de la présence des indicateurs non financiers comme moyen d'augmenter la performance, mais jamais de tester toutes les explications. Selon Ittner, Larcker et Randall (2003), il n'existe pas d'étude testant si les outils faisant une large place aux indicateurs de performance non financiers (comme le Balanced Scorecard) ont effectivement engendré une performance supérieure (p. 721). Leur approche présente cependant des limites. En effet, le Q de Tobin est utilisé en variable de contrôle alors qu'il s'agit d'un indicateur de performance, la variable dépendante représente toujours des mesures ex-post de la performance et enfin, ils ne considèrent pas d'autres variables de contrôle qui peuvent également expliquer la performance. Perera, Harrisson et Poole (1997) testent si la cohérence entre la stratégie axée sur le client et la présence d'indicateurs non financiers entraîne une performance supérieure en ne se focalisant que sur des mesures comptables (taux de croissance annuel des ventes, ROI, Return on Investments; ROE, Return on Equities). Ils privilégient des indicateurs de performance à court terme ce qui peut être contradictoire avec la présence des indicateurs non financiers. L'accroissement de la performance ne semble pas évidente pour Ittner et Larcker (1997) qui ajoutent la performance perçue comme variable dépendante. En revanche, Chenhall (1997), qui relie également la présence des indicateurs non financiers au système incitatif, constate une augmentation significative de la performance. Notons que ces résultats divergents peuvent vraisemblablement être expliqués selon qu'ils se focalisent sur une composante de la structure organisationnelle ou sur l'ensemble pour considérer que la cohérence entre la structure organisationnelle et la présence des indicateurs non financiers engendrera une plus grande performance. Autrement dit, le fait d'intégrer ou non l'hypothèse d'interdépendance entre les systèmes de contrôle, d'évaluation et d'incitation (hypothèse corroborée dans Demers (2002)) peut expliquer cette différence. De plus, les approches cognitives sont peu évoquées dans ces tests. Enfin, une réflexion s'impose afin de savoir si l'introduction des indicateurs non financiers selon l'approche cognitive notamment est censée améliorer la valeur actionnariale ou partenariale. L'ensemble de ces considérations ne remettent pas en question la méthode utilisée dans les analyses existantes (le 
recours aux questionnaires en l'occurrence) mais nous encouragent à mener une étude approfondie sur le choix des items.

\section{Bibliographie}

Anthony R.N. (1965), Planning and Control Systems: A Framework for Analysis, Division of Research, Graduate School of Business Administration, Harvard University, Boston, Mass.

Bourguignon A., Mallerret V., Norrekit H. (2002), «L'irréductible dimension culturelle des instruments de gestion : l'exemple du tableau de bord et du balanced scorecard », Comptabilité, Contrôle, Audit, mai, p.732.

Brickley J.A, Smith C.W, Zimmerman J.L. (1997), Managerial Economics and Organizational Architecture, McGraw-Hill.

Burns J., Scapens R.W. (2000), «Conceptualizing Management Accounting Change: An Institutional Framework », Management Accounting Research, vol.11, p. 3-25.

Charreaux G., 2002 (a) , «Quelle théorie pour la gouvernance ? De la gouvernance partenariale à la gouvernance cognitive », cahier de recherche, Fargo.

Charreaux G., 2002 (b) , «Variation sur le thème : A la recherche de nouvelles fondations pour la finance et la gouvernance d'entreprise », Finance Contrôle Stratégie, vol.5, n³, p. 5-68.

Charreaux G. et Desbrières P. (1998), «Valeur partenariale contre valeur actionnariale », Finance Contrôle Stratégie, vol. $1, n^{\circ} 2$, p. $57-88$.

Chenhall R.H. (1997), « Reliance on Manufacturing Performance Measure, Total Quality Management and Organizational Performance », Management Accounting Research, vol.8, p. 187-206.

Cornell B., Shapiro A.C. (1987), « Corporate Stakeholders and Corporate Finance », Financial Management, vol. $16, n^{\circ} 1$, p. $5-14$.

Cyert R.M. et March J.G. (1963), A Behavioral Theory of the Firm, Prentice Hall.

Demers E., Shachkell M., Widener S.K. (2002), « Complementarities in Organizational Design: Empirical Evidence from the New Economy », working paper, $54 \mathrm{p}$.

Edvinsson L. et Malone M. (1999), Le Capital Immatériel de l'Entreprise : Identification, Mesure, Management, Maxima, Laurent de Mesnil Editions. 
Edvinsson L., Sullivan P. (1996), « Developing a Model for Managing Intellectual Capital », European Management Journal, vol.14, n4, p. 356-364.

Feltham G., Xie J. (1994), « Performance Measure Congruity and Diversity in Multi-Task Principal/agent Relations », The Accounting Review, vol.69, p. 429-453.

Fullerton R.R., McWatters C.S (2002), « The Role of Performance Measures and Incentives Relation to the Degree of JIT Implementation », Accounting, Organizations and Society, vol. 27, p. 711-735.

Govindarajan V., Gupta A. (1985), « Linking Control Systems to Business Unit Strategy: Impact on Performance », Accounting, Organizations and Society, vol. 10, n 1, p. 51-66.

Granovetter M. (1994), «Les institutions économiques comme constructions sociales, un cadre d'analyses », in Analyse économique des conventions, Paris, PUF.

Hamel G. et Prahalad C.K. (1990), «The Core Competence of the Corporation », Harvard Business Review, vol. $68, \mathrm{n}^{\circ} 3, \mathrm{p} .79-92$.

Horngren C., Bhimani A., Datar S., Foster G. (2003), Contrôle de gestion et gestion financière, Pearson Education, $2^{\text {ème }}$ édition.

Ittner C.D., Larcker D.F., Randall T. (2003), « Performance Implications of Strategic Performance Measurement in Financial Services Firms », Accounting, Organizations and Society, vol. 28, p. 715-74.

Ittner C.D, Larcker D.F. (2002), « Determinants of Performance Measure Choices in Worker Incentives Plans », Journal of Labor Economics, vol. 20, n² 2, p. 58-90.

Ittner C.D, Larcker D.F. (1997), « Mutuality Strategy, Strategic Control Systems, and Organizational Performance », Accounting, Organization and Society, vol. 22, n 3-4, p. 293-314.

Ittner C.D, Larcker D.F., Rajan M.V (1997), « The Choice of Performance Measures in Annual Bonus Contracts », The Accounting Review, vol. 72, n², p. 231-255.

Jensen M.C, Meckling W.H. (1998), « Divisional Performance Measurement », in M.C Jensen, Foundations of Organizational Strategy, Harvard University Press.

Jensen M.C, Meckling W.H, (1992), « Specific and General Knowledge and Organizational Structure », Contract Economics, ed. Lars Werin and Hans Wijkander in M.C Jensen, Foundations of Organizational Strategy, 1998, Harvard University Press.

Jensen M.C, Meckling W.H. (1976), «Theory of the Firm : Managerial Behavior, Agency Costs and Ownership Structure », Journal of Financial Economics, vol. 3, n 4, p. 305-360. 
Johnson H.T. et Kaplan R.S. (1987), « Relevance Lost: The Rise and Fall of Management Accounting Systems », Harvard Business School Press, Boston.

Kaplan R.S. et Norton D.P. (2004), « Strategy Maps: Converting Intangible Assets into Tangible Outcomes », Harvard Business School Press, Boston.

Kaplan R.S. et Norton D.P. (2001), Comment utiliser le tableau de bord prospectif, Editions d'Organisation, Paris.

Kaplan R.S. et Norton D. (1998), Le Tableau de Bord Prospectif, Les Editions d'Organisation, Paris.

Langevin P. (1999), «Contrôle de gestion et théorie économique des organisations », in Y. Dupuy, Faire de la recherche en contrôle de gestion? De la compréhension des pratiques à un renouvellement théorique, Vuibert Fnege.

Laroche H. et Nioche J.P. (1998), Repenser la stratégie, fondements et perspectives, Vuibert, Paris.

March J.G. et Simon H.A. (1958), Organizations, Wiley and Sons, New York.

Mouritsen J. (1998), « Driving Growth : Economic Value Added versus Intellectual Capital », Management Accounting Research, vol. 9, p. 461-482.

Nelson R. et Winter S. (1982), An Evolutionary of Economic Change, Belnap Press of Harvard, University Press, Cambridge.

Nonaka I., Takeuchi H. (1997), La connaissance créatrice : la dynamique de l'entreprise apprenante, de Boeck Université.

Ouchi W.G. (1979), «A Conceptual Framework for the Design of Organizational Control Mechanisms », Management Science, vol. 25, $\mathrm{n}^{\circ}$ 9, p. 833-848.

Perera S., Harrison G., Poole M. (1997), « Customer-focused Manufacturing Strategy and the Use of Operationsbased Non-financial Performance Measures: A Research Note », Accounting Organizations and Society, vol. $22, \mathrm{n}^{\circ} 6, \mathrm{p} .557-572$

Porter M. (1985), Competitive Advantage: Creating and Sustaining Superior Performance, The Free Press, New York.

Roos J. (1998), « Exploring the Concept of Intellectual Capital», Long Range Planning, vol. 31, $\mathrm{n}^{\circ}$ 1, p. 150153.

Simon H.A. (1947), Administrative Behavior, a Study of Decision Making Processes in Administrative Organizations, Free Press, New York.

Simons R.L. (1995), Levers of Control, Harvard Business School Press, Boston. 
Simons R. (2000), Performance Measurement and Control Systems for Implementing Strategy, Texts and Cases, Upper Saddle River, Prentice Hall.

Vaivio J. (2004), « Mobilizing Local Knowledge with ' Provocative ' Non-financial Measures », European Accounting Review, vol. 13, n 1, p. 39-71.

Vaivio J. (1999), «Exploring a Non-financial Management Accounting Change », Management Accounting Research, vol. 10, p. 409-437.

\section{Annexe 1 \\ Utilisation de critères non financiers dans une logique contractuelle}

Si nous étudions attentivement certaines applications (Kaplan et Norton, 1998 et 2001 ; Simons, 2000) nous constatons que pour l'essentiel, les Balanced Scorecard déployés dans les entreprises américaines s'inscrivent dans la logique contractuelle que nous avons présentée.

Chez National Insurance (Kaplan et Norton, 1998, p. 163-169), compagnie américaine d'assurance-dommages, les seuls acteurs du processus de mise en place du Balanced Scorecard évoqués continuellement sont les dirigeants. Ce sont eux qui ont identifié les perspectives stratégiques, les objectifs stratégiques, les déterminants de la performance (indicateurs a priori), les mesures de résultats (indicateurs a posteriori), ainsi que les chaînes de causalité entre perspectives, objectifs et indicateurs. La stratégie est au préalable «clairement articulée et acceptée », le Balanced Scorecard ayant pour objectif de la «...clarifier et de la redéfinir...» ainsi que de «...tester les hypothèses sur lesquelles repose la stratégie...» (1998, p. 170). La mise en place du Balanced Scorecard a été rapide et s'est accompagnée de profonds changements au niveau des processus organisationnels ${ }^{13}$. Elle a été combinée à une réorientation stratégique et à un processus de reengineering (1998, p. 238). En étudiant cette fois l'exemple de la société d'assurances américaine Cigna (Kaplan et Norton, 2001), nous constatons les mêmes caractéristiques. La démarche a pour objectif de permettre la déclinaison d'une stratégie formulée ${ }^{14}$ avec précision et partagée par tous ainsi qu'une validation de ses hypothèses (2001, p. 17). A cette fin, Kaplan et Norton expliquent 
(2001, p. 16) que la stratégie est reliée au processus budgétaire et que des tests de corrélations statistiques entre les indicateurs du Balanced Scorecard sont développés afin de voir si par exemple les programmes de responsabilisation des salariés accroissent la satisfaction des clients et améliorent les processus. L'utilisation de «cartes stratégiques » constitue le support à la déclinaison de la stratégie (2001, p. 80-81). Kaplan et Norton précisent que « la structure du Balanced Scorecard a une logique verticale allant de haut en bas, en débutant avec les résultats souhaités sur le plan financier et celui du client... » (2001, p. 233). Concrètement, la stratégie est déclinée en quatre thèmes, l'objectif final à cinq ans étant de faire baisser le ratio combiné dépenses/recettes ${ }^{15}$. Ces quatre thèmes sont : gérer les agents (éliminer les mauvais et former les bons), se concentrer géographiquement, améliorer le processus de souscription (mettre l'accent sur la sélection au bon prix et former le personnel), et mettre en adéquation la souscription, les sinistres, le contrôle des pertes et les audits de primes.

Le déploiement du Balanced Scorecard de Cigna s'accompagne de processus de communication analogues à ceux utilisés pour le lancement de nouveaux produits. La communication facilite l'alignement stratégique et emporte l'adhésion du personnel. $70 \%$ du personnel a accès au Balanced Scorecard (2001, p. 344). Une fois le système mis en place, tous les autres documents ont été supprimés (2001, p. 349). Un système informatique complexe a rapidement été développé pour accompagner la mise en place du Balanced Scorecard (2001, p. 350).

Chez Cigna, les rémunérations au mérite des responsables ont été calculées dès la première année à partir d'indicateurs du Balanced Scorecard (2001, p. 19). Par la suite, les salariés se sont vus attribuer des primes en fonction d'un calcul fondé sur 14 indicateurs du Balanced Scorecard ${ }^{16}$.

\section{Annexe 2}

\section{Utilisation de critères non financiers dans une logique cognitive}


Edvinsson et Malone (1999) proposent un indice synthétique de mesure du Capital Intellectuel en prenant appui sur le Navigateur développé au sein de Skandia. Ils proposent la formule suivante pour calculer cet indice du Capital Intellectuel :

Capital Intellectuel $=\mathbf{i} \mathbf{x} \mathbf{C}$ avec,

- C : agrégation d'une vingtaine d'indicateurs exprimés en valeur absolue et orientés vers le développement à venir de la firme. Voici les neuf critères génériques qui sous-tendent ces indicateurs.

\section{Les revenus générés par de nouveaux produits :}

1- CA généré par de nouvelles opérations commerciales (nouveaux programmes/services).

\section{L'investissement dans de nouveaux canaux :}

2- investissements pour le développement de nouveaux marchés (consommateur/client/curriculum),

3- investissements pour le développement du secteur d'activité,

4- investissements pour le développement de nouveaux circuits.

\section{Le changement dans le stock de technologies de l'information :}

5- investissements informatiques consacrés aux ventes, au service et à l'assistance,

6- investissements informatiques consacrés à l'administration,

7- évolutions du parc informatique.

\section{L'investissement dans les ventes, le service et le soutien :}

8- investissements consacrés à l'assistance au consommateur (client),

9- investissements consacrés au service au consommateur (client),

10- investissements consacrés à la formation du consommateur,

11- frais par client non liés au produit.

\section{L'investissement dans le développement de ressources humaines :}

12- investissements consacrés par salarié au développement des compétences.

\section{La formation de personnel :}

13- investissements consacrés par salarié à la formation et à l'assistance pour les nouveaux produits, 
14- formations réservées aux salariés non basés dans l'entreprise,

15- investissements consacrés à la formation, à la communication et à l'assistance à destination des salariés permanents à plein temps,

16- programmes de formation et d'assistance réservés aux salariés travaillant à temps plein dans le cadre d'un contrat à durée déterminée,

17- programmes de formation et d'assistance réservés aux salariés travaillant à temps partiel dans le cadre d'un contrat à durée déterminée.

\section{L'investissement en coopération inter firmes :}

18- investissements consacrés au développement des partenariats/joint ventures,

19- mises à jour des systèmes informatisés d'échange de données (EDI) ou des réseaux.

\section{L'investissement en marques :}

20- investissements consacrés à l'identification de la marque (logo/nom).

\section{L'investissement en nouveaux brevets, nouveaux copyrights :}

21- investissements consacrés aux brevets et aux droits de licence.

Cette liste privilégie les dépenses d'investissement et ne met l'accent que sur les capacités futures à dégager des profits. Or le capital immatériel recouvre également les capacités actuelles d'enrichissement. Un mauvais investissement ou un investissement mal géré est souvent pire qu'une absence d'investissement.

- Avec i, facteur compensatoire qui relativise la portée de ces investissements, on tient compte de la productivité réelle, de la création de valeur et de l'évaluation que fera l'utilisateur. i est le coefficient d'efficience de l'organisation dans l'utilisation du Capital Intellectuel, calculé à partir de neuf indicateurs clés établis en pourcentage et renvoyant aux différentes composantes du navigateur. Les auteurs proposent les indicateurs suivants : la part de marché (\%), l'indice de satisfaction des clients (\%), l'indice de leadership (\%), l'indice de motivation (\%), l'indice de ressources en recherche et développement (\%), l'indice d'heures de formation (\%), l'objectif de performance / qualité (\%), le taux de maintien des salariés (\%) et l'efficacité administrative/chiffre d'affaires (inverse du ratio erreur administrative/chiffre d'affaires) (\%). 
${ }^{1}$ Ce sont par exemple des indicateurs quant à la part de marché ou le nombre de nouveaux clients, des indicateurs de productivité, des indices de satisfaction des clients ou des salariés.... Cela peut être des indicateurs qualitatifs ou quantitatifs parfois exprimés en valeur monétaire (comme les dépenses en programmes de formation par salarié, frais de marketing par client). Ils sont non financiers car ils n'expriment pas directement l'objectif financier de la firme comme peuvent le faire des indicateurs de rentabilité, fondés sur le résultat ou le chiffre d'affaires.

${ }^{2}$ C'est pourquoi nous excluons de notre champ d'investigation des grilles de lectures théoriques telles que la théorie des conventions. Cette théorie repose en effet sur le principe que les comportements mimétiques dans les organisations sont les déterminants principaux des choix managériaux. Or, nous partons au contraire de l'idée que la recherche de la création de valeur conditionne pour l'essentiel les décisions prises dans les organisations. Ce positionnement n'exclut cependant pas que nous puissions considérer que les comportements mimétiques constituent un facteur, mais parmi bien d'autres, qui détermine ces décisions.

${ }^{3}$ Par courant conventionnel, nous entendons des grilles d'analyse stratégiques de nature concurrentielle, c'est-àdire des grilles de lectures où les facteurs relatifs à l'environnement concurrentiel jouent un rôle prépondérant : il s'agit par exemple des matrices stratégiques du Boston Consulting Group ou encore du modèle des cinq forces de Porter. Le Mouvement des Ressources et des Compétences constitue une grille d'analyse en rupture avec ce courant conventionnel.

${ }^{4}$ Des illustrations de Balanced Scorecard sont fournies dans l'annexe 1.

${ }^{5}$ Un exemple d'utilisation de cet outil est fourni en annexe.

${ }^{6}$ Par création de connaissances organisationnelles, nous entendons la capacité d'une entreprise dans son ensemble à créer de nouvelles connaissances, à les diffuser en son sein à et à les incorporer dans ses produits, services et systèmes (p. 21).

${ }^{7}$ Edvinsson et Malone (1999) expliquent en substance que le Capital Structurel est ce qu'il reste dans l'entreprise lorsque les salariés rentrent chez eux le soir.

${ }^{8}$ Ils dissocient les actifs intellectuels pouvant et devant être protégés au risque d'être imités par la concurrence, de la connaissance tacite ou inobservable, intrinsèquement protégée, parce que difficilement transférable.

${ }^{9}$ Voir notamment les outils de simulation Tango et Mango développés par Celemi (http://www.Celemi.com). Celemi est une société suédoise spécialisée dans l'aide au déploiement de tableaux de bord centrés sur des indicateurs de pilotage du capital intellectuel.

${ }^{10}$ Etude de cas exploratoire dans une entreprise britannique du secteur de la chimie.

${ }^{11}$ Il évoque alors la mise en place d'une démarche de Management par la Qualité Totale.

${ }^{12}$ C'est-à-dire en se situant davantage dans une perspective psychologique que sociologique.

${ }^{13}$ Kaplan et Norton précisent (p. 170, 1998) que «...la démarche de mesure a bel et bien servi à piloter le changement ».

${ }^{14}$ Cette vocation d' « alignement stratégique » de la démarche a été encore réaffirmée dernièrement (Kaplan et Norton, 2004 b, p. 32).

${ }^{15}$ Règlement des sinistres et frais d'exploitation /primes.

${ }^{16} \mathrm{Ce}$ calcul prend en compte la performance individuelle du salarié ainsi que la performance de son centre de responsabilité. 